\title{
WING: A Web Probing, Visualization, and Performance Analysis Service
}

\author{
Leszek Borzemski and Ziemowit Nowak \\ Wroclaw University of Technology \\ Wybrzeze Wyspianskiego 27 \\ 50-370 Wroclaw, Poland \\ \{leszek.borzemski; ziemowit.nowak\}@pwr.wroc.pl
}

\begin{abstract}
An open network service Wing has been developed for the purpose of Web probing, visualization and performance analysis from the user perspective. Wing downloads target page to the service location and returns to the user a page showing HTTP timeline chart and both detailed and aggregated information about web page downloading. Contrary to other systems that use their own Web clients developed for particular visualization tools only, Wing supports real-life studies as it uses a real Web browser. Wing employs all features of HTTP/1.1 and can process scripts so we can automate the usage of the service and apply it to the advanced Internet measurements.
\end{abstract}

\section{Introduction}

Along with the development of computer networks, network protocol visualization tools have been designed. They are used for better understanding of computer network. Nowadays the most important visualization challenge is HTTP protocol, and especially Web page downloading. Both end-users and site administrators are anxious to get knowledge about the downloading using different browsers. We present Wing, a network service for Web probing, visualization and performance analysis from the user perspective.

\section{Web Page Measurement and Visualization}

Passive and active measurement techniques can be used for visualization of Web transactions. Most of them are active probing projects that are based on the target Web page measurement employing a special measuring tool. Unfortunately, to the authors' knowledge available such network services use their own Web browsing methods. Using specially developed browsers does not ensure that we get adequate results. Probably the MyKeynote [2] is the most advanced benchmarking service that measures Web site's performance and its availability from a word-wide network of measurement agents. Unfortunately, the MyKenote client uses HTTP/1.0 protocol that is not common in the Internet now. Leading browsers, such as MS Internet Explorer, 
use more advanced HTTP/1.1 protocol. Since the browsers download Web pages in different ways giving different results, it appears necessary to develop tools supporting real-life studies.

\section{Wing Service}

The Wing (Web ping) Internet service has been developed for the purpose of Web probing, visualization and performance analysis from the user perspective. Our service gathers, stores in a database and visualizes data about the real Web browser's activity. Today's implementation is done for MS IE but our service can monitor activity of any browser. It employs all features of HTTP/1.1 and can process scripts so we can automate the usage of the service and apply it to the advanced Internet measurements.

Wing is activated by a remote user request sent to Wing controller targeting URL to be tested. As the service is invoked, the Wing controller calls a local Web client that issues GET request to target URL as well as GET requests for all objects that are embedded in a target page. Wing monitors and time-stamps all browser's activities, determines the end of web page downloading and preprocesses gathered data into the format convenient for further analysis, as well as for visualization. Data is stored in a database server. Wing returns to the remote user a web page visualizing how the target Web page has been loaded locally. The return page shows HTTP timeline chart and both detailed and aggregated information about web page downloading.

Wing supports IP, TCP, UDP, DNS and HTTP protocols, logging a dozen parameters of HTTP transactions and TCP connections, thus facilitating a much deeper analysis. For example, we used it in the research on the estimation of the HTTP throughput and TCP Round-Trip Time (RTT) on Internet link between a Web browser and World-Wide Web server [1]. Wing estimated the RTT based on the measurements of time spacing between the SYN packet sent by the client and the SYN-ACK packet received by the client.

\section{Conclusions}

Our free network service has the ability to allow visualization of the real-life Web browsers including MS IE and Linux based solutions. It can periodically gather data for further off-line analysis, not only for the ad hoc visualization needs. Thus we can monitor target Web page downloading behavior over demanded period of time.

\section{References}

1. Borzemski L., Nowak Z.: Estimation of HTTP throughput and TCP Round-Trip Times, Proceedings of 10th Polish Teletraffic Symposium, IEEE Chapter, Cracow (2003) 335-352

2. http://www.mykeynote.com 